\title{
Potential application of genomic profiling for the diagnosis and treatment of patients with sarcoma
}

\author{
LIBIN XU $^{1 *}$, XIANBIAO XIE ${ }^{2 *}$, XIAOLIANG SHI ${ }^{3}$, PENG ZHANG $^{3}$, ANGEN LIU $^{3}$, JIAN WANG ${ }^{4}$ and BO ZHANG ${ }^{5}$ \\ ${ }^{1}$ Department of Orthopedic Surgery, National Cancer Center/National Clinical Research Center for Cancer/Cancer Hospital, \\ Chinese Academy of Medical Sciences and Peking Union Medical College, Beijing 100021; ${ }^{2}$ Department of \\ Musculoskeletal Oncology, The First Affiliated Hospital of Sun Yat Sen University, Guangzhou, Guangdong 510080; \\ ${ }^{3}$ OrigiMed Co. Ltd., Shanghai 201114; ${ }^{4}$ Department of Pathology, Fudan University Shanghai Cancer Center, \\ Shanghai 200032; ${ }^{5}$ Department of Pathology, Peking University Third Hospital, Beijing 100191, P.R. China
}

Received March 31, 2020; Accepted December 14, 2020

DOI: $10.3892 / \mathrm{ol} .2021 .12614$

\begin{abstract}
Sarcomas represent a heterogeneous group of mesenchymal malignancies arising at various locations in the soft tissue and bone. Though a rare disease, sarcoma affects $\sim 200,000$ patients worldwide every year. The prognosis of patients with sarcoma is poor, and targeted therapy options are limited; therefore, accurate diagnosis and classification are essential for effective treatment. Sarcoma samples were acquired from 199 patients, in which TP53 (39.70\%, 79/199), CDKN2A (19.10\%, 38/199), CDKN2B (15.08\%, 30/199), KIT (14.07\%, 28/199), ATRX (10.05\%, 20/199) and RB1 (10.05\%, 20/199) were identified as the most commonly mutated genes ( $>10 \%$ incidence). Among 64 soft-tissue sarcomas that were unclassified by immunohistochemistry, 15 (23.44\%, 15/64) were subsequently classified using next-generation sequencing (NGS). For the most part, the sarcoma subtypes were evenly distributed between male and female patients,
\end{abstract}

Correspondence to: Professor Bo Zhang, Department of Pathology, Peking University Third Hospital, 49 Huayuan Road, Haidian, Beijing 100191, P.R. China

E-mail: zhanbo@bjmu.edu.cn

Professor Jian Wang, Department of Pathology, Fudan University Shanghai Cancer Center, 270 Dongan Road, Xuhui, Shanghai 200032, P.R. China

E-mail: softtissuetumor@163.com

*Contributed equally

Abbreviations: NGS, next-generation sequencing; FISH, fluorescence in situ hybridization; GA, genomic alteration; GIST, gastrointestinal stromal tumor; SNV, single nucleotide variant; Indel, insertion-deletion polymorphism; $\mathrm{CNV}$, copy number variation; TMB, tumor mutational burden; DFSP, dermatofibrosarcoma protuberans; TMB-H, high tumor mutational burden; ICPI, immune checkpoint inhibitor

Key words: sarcoma, diagnosis, next-generation sequencing, genomic profiling, tumor mutational burden, biomarker while a significant association with sex was detected in leiomyosarcomas. Statistical analysis showed that osteosarcoma, Ewing's sarcoma, gastrointestinal stromal tumors and liposarcoma were all significantly associated with the patient age, and that angiosarcoma was significantly associated with high tumor mutational burden. Furthermore, serially mutated genes associated with myxofibrosarcoma, gastrointestinal stromal tumor, osteosarcoma, liposarcoma, leiomyosarcoma, synovial sarcoma and Ewing's sarcoma were identified, as well as neurotrophic tropomyosin-related kinase (NTRK) fusions of IRF2BP2-NTRK1, MEF2A-NTRK3 and ITFG1-NTRK3. Collectively, the results of the present study suggest that NGS-targeting provides potential new biomarkers for sarcoma diagnosis, and may guide more precise therapeutic strategies for patients with bone and soft-tissue sarcomas.

\section{Introduction}

Sarcoma is a rare malignant tumor that frequently occurs in, or originates from, the bone, cartilage or connective tissue (1). Globally, almost 200,000 patients are affected by sarcoma each year (2). The prognosis of patients is poor, and the choice of approved targeted drugs is somewhat limited $(3,4)$. Surgery is currently the primary treatment option for most sarcomas, but local recurrence does occur (5). Targeted molecular therapies have yielded improved clinical outcomes (6-8). However, due to diagnostic difficulties, bone and soft-tissue sarcomas are often only diagnosed at the advanced stage, resulting in a $50-60 \%$ 5-year survival rate $(9,10)$. Therefore, a more accurate system for sarcoma diagnosis and classification is urgently required.

Sarcoma includes soft-tissue sarcomas and primary bone sarcomas (11). Soft-tissue sarcomas comprise $>50$ subtypes (12), and the most frequently observed subtypes include liposarcoma, leiomyosarcoma, undifferentiated soft-tissue sarcoma, fibrosarcoma and synovial sarcoma (13). Primary bone sarcoma subtypes include Ewing's sarcoma and osteosarcoma (14). Histopathological examination, such as the analysis of histological sections and fluorescence in situ hybridization (FISH), are still the only available methods for the accurate diagnosis of sarcomas. However, due to their 
rarity and diversity, the classification of sarcomas remains a challenge. The identification and application of potential biomarkers is a convenient, rapid and accurate strategy for identifying sarcoma subtypes, and is conducive to improving diagnosis and prognostic prediction (15-18).

With continuous developments in molecular biology, next-generation sequencing (NGS) technology has enabled more accurate and efficient molecular characterization. The Cancer Genome Atlas and the International Cancer Genome Consortium have characterized the genome and genomic alterations (GAs) of most types of cancer $(19,20)$, and a number of recent studies have focused on the molecular profiling of sarcomas $(21,22)$. Sarcomas can be divided into the following subgroups according to genetic heterogeneity: i) Gene fusions; ii) genomic amplifications; and iii) extensive combinations of genomic imbalances and point mutations (23-25). There is also evidence to suggest that some sarcomas possess unique molecular characteristics, such as the SYT-SSX fusion in synovial sarcoma, the $E W S$-ATF1 fusion in clear cell sarcoma, and the EWSR1-FLII fusion in Ewing's sarcoma (26-28). Specific molecular characterizations not only assistant in the classification of sarcoma, but can also guide treatment programs. For example, imatinib has demonstrated good efficacy in gastrointestinal stromal tumor (GIST) patients with KIT, PDGFRA, CSF1 and $A B L$ mutations (29-31); since 2013, palbociclib has undergone phase II clinical trials in liposarcoma patients with CDK4 mutations (32). Therefore, a clear classification system and a precise molecular description of sarcoma subtypes are necessary for subsequent diagnosis and treatment.

The present study aimed to identify GAs for the mutational profiling of 199 patients with sarcoma (both soft-tissue and primary bone sarcoma). By comparing molecular-based classification with traditional immunohistochemical categorization, the accuracy and necessity of NGS technology for sarcomas classification was confirmed. The results provide comprehensive and accurate information of GAs, which suggest novel biomarkers for sarcoma diagnosis that may guide precise therapeutic strategies for patients with bone and soft-tissue sarcomas.

\section{Materials and methods}

Patient enrollment and sample collection. The present study was approved by the Ethics Committees of National Cancer Center/Cancer Hospital, Chinese Academy of Medical Sciences and Peking Union Medical College (Beijing, China) and the First Affiliated Hospital of Sun Yat Sen University (Guangzhou, China). A total of 199 patients with sarcoma were enrolled between January 1, 2008 and December 31, 2018. Tumor tissues were collected from patients, fixed in formalin and embedded in paraffin. Matched blood samples were collected as controls for GA detection.

Identification of GAs and measurement of tumor mutational burden (TMB). Total DNA was obtained from both formalin-fixed paraffin-embedded (FFPE) tumor tissues and matched blood samples of each patient using the QIAamp DNA FFPE Tissue Kit and QIAamp DNA Blood Midi Kit (both Qiagen $\mathrm{GmbH}$ ), respectively. The DNA samples were sequenced using the next-generation sequencing-based
YuanSu450 ${ }^{\mathrm{TM}}$ gene panel $\left(\right.$ OrigiMed $\left.^{\circledR}\right)$, in a laboratory certified by the College of American Pathologists (CAP) and the Clinical Laboratory Improvement Amendments (CLIA). The genes were captured and sequenced with a mean depth of 800x using the NextSeq 500 system (Illumina, Inc.). Single nucleotide variants (SNVs) were identified using MuTect (v1.7, www.broadinstitute.org/cancer/CGA), and insertion-deletion polymorphisms (indels) were identified using PINDEL (V0.2.5, https://www.pindel.com). The functional impact of these mutations was annotated using SnpEff3.0 (http://snpeff. sourceforge.net). Copy number variation (CNV) regions were identified by Control-FREEC (v9.7, http://boevalab.inf. ethz.ch/FREEC/index.html.) with the following parameters: Window $=50,000$, and step $=10,000$. Gene fusions/rearrangements were detected using the following in-house pipeline: Paired-end reads with an abnormal insert size of $>2,000 \mathrm{bp}$ (aligned to the same or different chromosomes) were collected; discordant read pairs were clustered according to the pairing relationship, and consistent breakpoints from the paired-end discordant reads (within a single cluster) were identified to establish potential fusion/rearrangement breakpoints. Gene fusion/rearrangements were assessed using the Integrative Genomics Viewer (v2.4, http://software.broadinstitute. org/software/igv/ReleaseNotes/2.4.x). The TMB of each patient was calculated by counting the number of somatic mutations (including SNVs and indels) per megabase $(\mathrm{Mb})$ of the sequence examined.

Statistical analysis. Statistical analyses were performed using SPSS version 22.0 (IBM Corp) and significant differences were detected using Fisher's exact test. $\mathrm{P}<0.05$ was considered to indicate a statistically significant difference.

\section{Results}

Clinical characteristics of patients with sarcoma. A total of 199 patients with soft-tissue or osteogenic sarcomas were enrolled in the present study. This included 105 male and 94 female patients, with a median age of 50 years (range, 1-86 years). The TMB values of all patients were identified, from which 197 valid values were obtained with a median of 1.5 muts/Mb (range, 0.7-24.5 muts/Mb) (Table I).

GAs in 199 patients with sarcoma. Based on NGS targeting of 450 cancer-associated genes, a total of 1,077 clinically relevant GAs were identified in 288 genes (Fig. 1), with an average of 5.41 alterations per sample (range, 0-21). Among these GAs, CNV was the most frequent mutation type (49.21\%, 530/1,077), followed by SNV/short indel (39.83\%, 429/1077), gene fusion $(7.99 \%, 86 / 1,077)$ and long indel $(2.97 \%, 32 / 1,077)$ (Fig. 1 and Table SI). The most commonly mutated genes with a mutation frequency of $>10 \%$ were TP53 (39.70\%, 79/199), CDKN2A (19.10\%, 38/199), CDKN2B (15.08\%, 30/199), KIT $(14.07 \%, 28 / 199), \operatorname{ATRX}(10.05 \%, 20 / 199)$ and $R B 1(10.05 \%$, 20/199). Notably, most mutations in TP53, KIT and ATRX were SNVs, while those in $C D K N 2 A, C D K N 2 B$ and $R B 1$ were CNVs (Fig. 2).

NGS aids the diagnosis of sarcoma. All sarcomas were pathologically diagnosed before sample collection, after 
Table I. Clinicopathological features of 199 patients in the sarcoma cohort.

\begin{tabular}{lc}
\hline Variable & Value \\
\hline Sex, n (\%) & \\
Male & $105(52.76)$ \\
Female & $94(47.24)$ \\
Median age (range), years & $50(1-86)$ \\
Median TMB (range), muts/Mb & $1.5(0.7-42.5)$ \\
\hline
\end{tabular}

TMB, Tumor mutational burden.

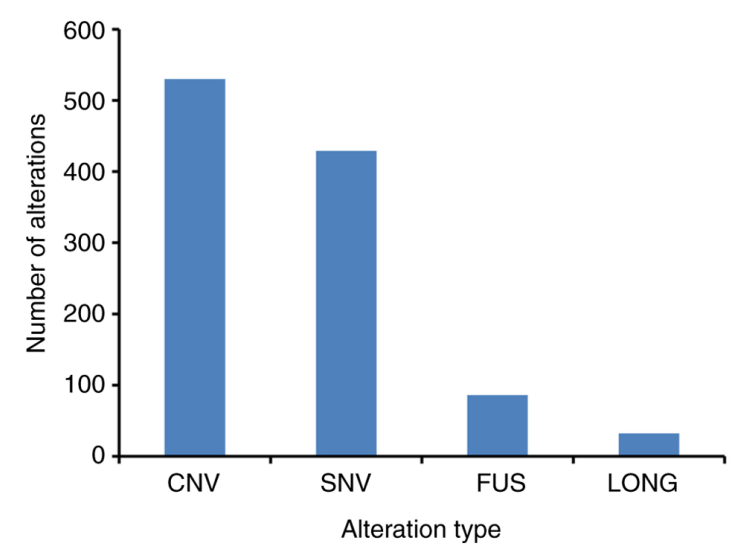

Figure 1. Statistical distribution map of variation types. CNV, copy number variations; SNV, single nucleotide variants; FUS, gene fusion; LONG, long insertion-deletion polymorphism.

which an experienced pathologist was invited to make a second diagnosis. Only those with the same diagnostic results were considered to be classified, while those with inconsistent or undetermined diagnostic results were considered as unclassified. As a result, 23 GISTs, 22 osteosarcomas, 12 myxofibrosarcoma, 11 liposarcomas, 11 leiomyosarcomas, 9 synovial sarcomas, 5 chondrosarcomas, 5 aggressive fibromatosis, 5 rhabdomyosarcomas, 4 Ewing's sarcomas, 4 angiosarcomas, 4 undefferentiated pleomorphic sarcomas, 3 mesotheliomas, 2 epithelioid hemangioendotheliomas, 2 myofibroblastic sarcomas, 2 myxoid sarcomas, 1 dermatofibrosarcoma protuberan, 1 solitary fibrous tumor, 1 embryonic undifferentiated sarcoma, 1 alveolar soft part sarcoma, 1 clear cell sarcoma, 1 malignant granular cell tumor, 1 myolipoma, 64 unclassified soft-tissue sarcomas and 4 unclassified osteogenic sarcomas were identified (Table II). Therefore, further classification was carried out according to the results of NGS.

According to the NGS detection results, 15 additional sarcoma cases were identified and classified, including 3 liposarcomas with amplifications in $M D M 2$ and $C D K 4,3$ Ewing's sarcomas with EWSR1 fusions, 3 dermatofibrosarcoma protuberans (DFSP) cases with fusions of $P D G F B$ (COL1A1-PDGFB), 2 leiomyosarcomas with mutations of $R B 1$ and TP53, 2 infantile fibrosarcomas with the fusion of ETV6-NTRK3, 1 GIST with a mutation in PDGFRA, and 1 $N T R K$ rearranged spindle cell mesenchymal tumor. Among them, 1 leiomyosarcoma was misdiagnosed as a GIST before
NGS auxiliary diagnosis. However, 54 cases remained unclassifiable. The primary characteristic mutations of these 15 sarcomas are listed in Table III.

Association of GAs with sarcoma subtypes. The mutational landscapes of sarcoma subtypes including GIST, osteosarcoma, liposarcoma, leiomyosarcoma and myxofibrosarcoma, were subsequently analyzed. The most common mutated genes in GISTs were KIT, CDKN2A and $C D K N 2 B$, and the most commonly mutated genes in osteosarcoma were $T P 53$, NCOR1, RB1, GID4, LRPIB, PTEN, ATRX, CCND3, MAP2K4 and RICTOR. In liposarcoma, CDK4, MDM2, FRS2, LRPI and TP53 were the most frequently mutated, as were TP53, MAP2K4, GID4, KDM6A and MCL1 in leiomyosarcoma. The most commonly mutated genes in myxofibrosarcoma were TP53, CDKN2A, CDKN2B, FAM135B, AKT2 and JUN (Fig. 3).

Statistical analysis revealed that mutations in TP53, AKT2, FAM135B, CDKN2A, JUN, CDKN2B, ROS1, AXL, SETD2 and $C C N E 1$ were significantly associated with myxofibrosarcoma (Table IV). The primary mutation type in GISTs was SNV, and mutations in KIT and TP53 were significantly associated with GISTs. Gene amplifications were the most common mutations in osteosarcoma and liposarcoma (Fig. 2). Mutations in NCOR1, GID4, LRPIB, RB1, AURKB, GLI2, RICTOR, MAP2K4, STK24, TNFSF13B, CCNE1, PRKDC, PTEN, CCND3, FGF10, BRD4, PRKACA, RET and IL7R were significantly associated with osteosarcoma, while those in CDK4, MDM2, FRS2, FUS, LRP1, MYB, PTPN11 and TYK2 were significantly associated with liposarcoma (Table IV). Furthermore, mutations in MAP2K4, TP53 and KDM6A were significantly associated with leiomyosarcoma (Table IV). Except for the negative association between TP53 mutations and GISTs, the majority of these frequently-mutated genes significantly occurred in corresponding sarcomas. Although only 9 synovial sarcomas and 7 Ewing's sarcomas were identified in the present study, the mutations in SS18 and EWSRI significantly occurred in synovial sarcoma and Ewing's sarcoma, respectively. Notably, the mutation of TP53 was also significantly negatively associated with synovial sarcoma (Table IV).

Association between TMB value, sarcoma subtype and patient demographics. The associations between sarcoma subtype, TMB value and patient sex and age were further analyzed. Based on age distribution, the patients we categorized into 4 groups: i) 1-19 years; ii) 20-39 years; iii) 40-59 years; iv) and 60-86 years of age. Osteosarcoma and Ewing's sarcoma commonly occurred in younger patients (1-19 years old), accounting for $40.91 \%(9 / 22)$ and $57.14 \%$ (4/7), respectively; GISTs and liposarcomas were more common in elderly patients (60-86 years old), accounting for 39.13\% (9/23) and $71.43 \%$ (10/14), respectively; and synovial sarcoma commonly occurred in young patients (20-39 years old), accounting for $66.67 \%$ (6/9). Statistical analysis showed that osteosarcomas, Ewing's sarcomas and synovial sarcomas significantly occurred in younger patients, while liposarcomas and GISTs significantly occurred in older patients (Fig. 4).

Most of the sarcoma subtypes had comparable frequencies in male and female patients, while 11 of the 13 leiomyosarcomas 


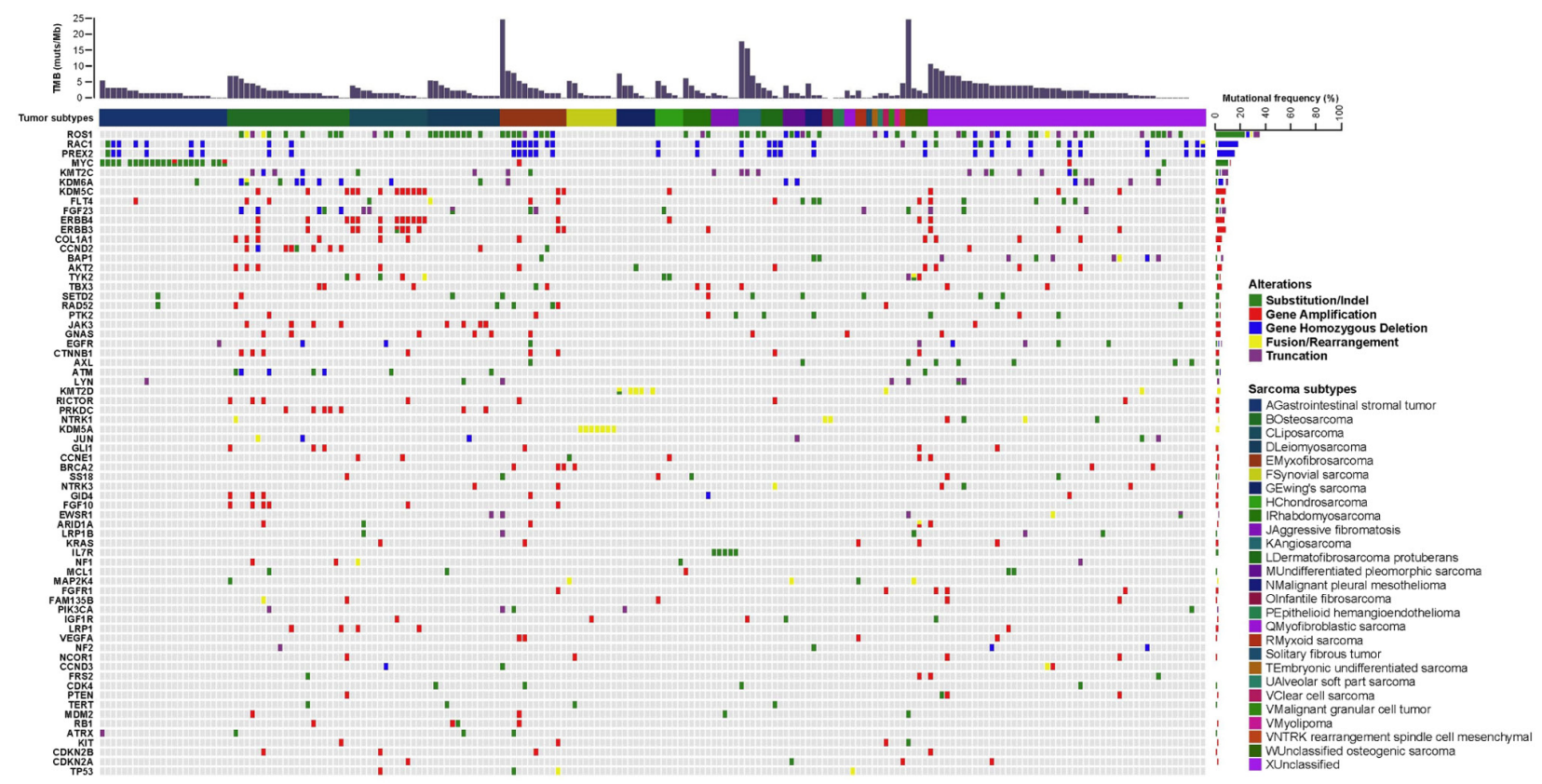

Figure 2. Mutational profiling of 199 patients with sarcoma. X-axis represents each case sample, and the Y-axis represents each mutated gene. Bar graphs to the right and above show the gene mutation frequency of each sample, and the TMB value of all samples, respectively. Green represents substitution/insertion-deletion polymorphism, red represents gene amplification, blue represents gene homozygous deletion, yellow represents fusion/rearrangement, and purple represents truncation. TMB, tumor mutation burden.
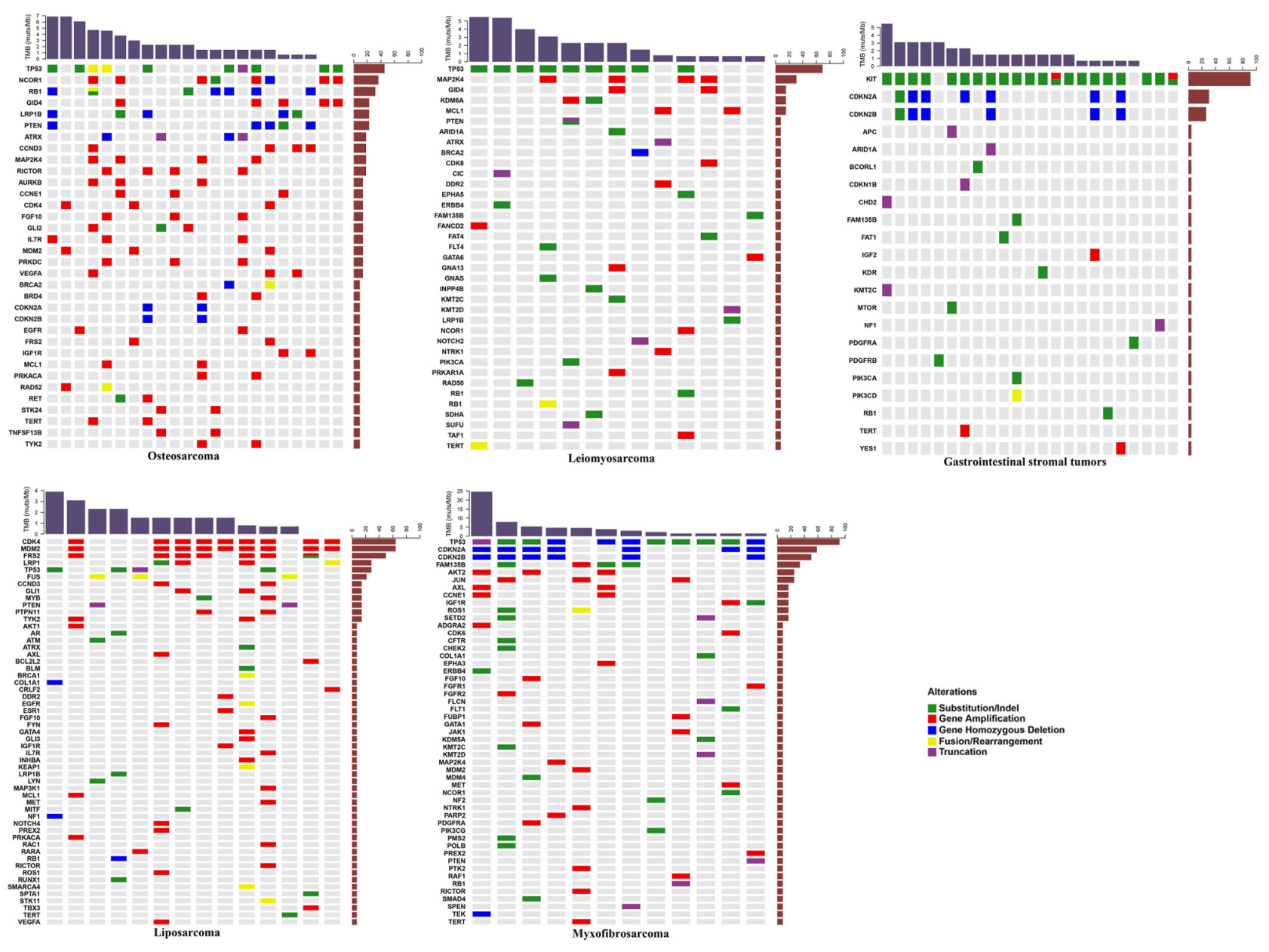

Figure 3. Mutational profiling of sarcoma subtypes. X-axes represent each case sample and the Y-axes represent the mutated genes. Bar graphs to the right and above show the gene mutation frequency of each sample, and the TMB value of each subtype, respectively. Green represents substitution/insertion-deletion polymorphism, red represents gene amplification, blue represents gene homozygous deletion, yellow represents fusion/rearrangement, and purple represents truncation. TMB, tumor mutation burden. 
Table II. Comparison of sarcoma subtypes identified by histochemistry- and NGS-based methods.

\begin{tabular}{|c|c|c|}
\hline Sarcoma subtype & Histochemistry-based, $\mathrm{n}$ & NGS-based, $\mathrm{n}$ \\
\hline Gastrointestinal stromal tumor & 23 & 23 \\
\hline Osteosarcoma & 22 & 22 \\
\hline Liposarcoma & 11 & 14 \\
\hline Leiomyosarcoma & 11 & 13 \\
\hline Myxofibrosarcoma & 12 & 12 \\
\hline Synovial sarcoma & 9 & 9 \\
\hline Ewing's sarcoma & 4 & 7 \\
\hline Chondrosarcoma & 5 & 5 \\
\hline Rhabdomyosarcoma & 5 & 5 \\
\hline Aggressive fibromatosis & 5 & 5 \\
\hline Angiosarcoma & 4 & 4 \\
\hline Dermatofibrosarcoma protuberans & 1 & 4 \\
\hline Undifferentiated pleomorphic sarcoma & 4 & 4 \\
\hline Malignant pleural mesothelioma & 3 & 3 \\
\hline Infantile fibrosarcoma & 0 & 2 \\
\hline Epithelioid hemangioendothelioma & 2 & 2 \\
\hline Myofibroblastic sarcoma & 2 & 2 \\
\hline Myxoid sarcoma & 2 & 2 \\
\hline Solitary fibrous tumor & 1 & 1 \\
\hline Embryonic undifferentiated sarcoma & 1 & 1 \\
\hline Alveolar soft part sarcoma & 1 & 1 \\
\hline Clear cell sarcoma & 1 & 1 \\
\hline Malignant granular cell tumor & 1 & 1 \\
\hline Myolipoma & 1 & 1 \\
\hline NTRK rearrangement spindle cell mesenchymal tumors & 0 & 1 \\
\hline Unclassified osteogenic sarcoma & 4 & 4 \\
\hline Unclassified & 64 & 50 \\
\hline
\end{tabular}

NGS, next-generation sequencing; NTRK, tropomyosin-related kinase.

occurred in females. Statistical analysis showed that leiomyosarcomas occurred significantly more often in women than in men (Fig. 5A). TMB values were obtained from 194 of the 199 enrolled patients. High TMB (TMB-H), which was defined as a TMB value $>10$ muts/Mb, was observed in 5 patients, including 1 with fibrosarcoma, 2 with angiosarcoma and 2 patients with unclassified soft-tissue sarcoma. Notably, only 4 angiosarcomas were identified in the cohort, and statistical analysis showed that angiosarcoma was significantly associated with TMB-H (Fig. 5B).

New neurotrophic tyrosine kinase (NTRK)1/3 fusions in the current cohort. NTRK $1 / 3$ mutations were detected in 11 of the 199 patients with sarcoma. Among these patients, 4 NTRK1 and 1 NTRK 3 mutations were gene amplifications, and 2 NTRK1 and 4 NTRK3 mutations were gene fusions, including 1 LMNA-NTRK1, 1 IRF2BP2-NTRK1, 1 MEF2A-NTRK3, 1 ITFG1-NTRK3 and 2 ETV6-NTRK3 fusions (Table V). Similar to previous reports $(33,34)$, gene fusions of ETV6-NTRK3 and LMNA-NTRK1 were detected in 2 infantile fibrosarcoma cases and 1 unclassified sarcoma, respectively. To the best of our knowledge, the present study is the first to describe the fusion of IRF2BP2-NTRK1, MEF2A-NTRK3 and ITFG1-NTRK3 in sarcoma. Therefore, sarcoma patients with $N T R K$ fusions or amplifications may potentially benefit from NTRK inhibitor therapy.

\section{Discussion}

The tumorigenesis of sarcoma is characterized by genomic abnormalities, manifested as multiple phenotypic changes and divided into various subtypes (35). To date, histological examination remains the primary method of sarcoma diagnosis (36). The histological and molecular heterogeneity of sarcoma make it particularly difficult to diagnose, though with the rapid development of NGS technology, increasing numbers of sarcoma genome sequencing studies have emerged (37-40). In the present study, the most commonly mutated genes were identified in 199 patients with sarcoma, and included TP53, CDKN2A, CDKN2B, KIT, ATRX and RB1. TP53 encodes the p53 protein and functions in the p53 pathway, while the $C D K N 2 B$ and $C D K N 2 A$ genes are associated with the regulation of p53 pathways (41). These findings suggest that p53 pathway mutations frequently occurred in the present cohort, 
Table III. List of cases diagnosed by next-generation sequencing.

\begin{tabular}{|c|c|c|c|}
\hline Case & Sarcoma subtype & Mutated genes & Mutation type \\
\hline 1 & DFSP & COL1A1-PDGFB & FUS \\
\hline 2 & DFSP & COL1A1-PDGFB & FUS \\
\hline 3 & DFSP & COLIA1-PDGFB & FUS \\
\hline 4 & Ewing's sarcoma & EWSR1 (EWSR1-FLII) & FUS \\
\hline 5 & Ewing's sarcoma & EWSR1 (EWSR1-ERG) & FUS \\
\hline 6 & Ewing's sarcoma & EWSRI (EWSR1-Intergenic) & FUS \\
\hline \multirow[t]{2}{*}{7} & Liposarcoma & $C D K 4$ & CNV \\
\hline & & $M D M 2$ & CNV \\
\hline \multirow[t]{2}{*}{8} & Liposarcoma & $C D K 4$ & CNV \\
\hline & & $M D M 2$ & CNV \\
\hline \multirow[t]{2}{*}{9} & Liposarcoma & $C D K 4$ & CNV \\
\hline & & $M D M 2$ & $\mathrm{CNV}$ \\
\hline \multirow[t]{2}{*}{10} & Leiomyosarcomas & $R B 1$ & SNV \\
\hline & & TP53 & SNV \\
\hline 11 & Leiomyosarcomas & TP53 & SNV \\
\hline 12 & Infantile fibrosarcoma & ETV6-NTRK3 & FUS \\
\hline 13 & Infantile fibrosarcoma & ETV6-NTRK3 & FUS \\
\hline 14 & GIST & PDGFRA & SNV \\
\hline 15 & Spindle cell mesenchymal tumor & NTRK1 (LMNA-NTRK1) & FUS \\
\hline
\end{tabular}

DFSP, dermatofibrosarcoma protuberans; GIST, Gastrointestinal stromal tumor FUS, fusion; CNV, copy number variant; SNV, single nucleotide variant.

which is consistent with a previous report (42). Somatic mutations of TP53 are associated with poor prognosis and low chemotherapy response rates in various tumor types $(43,44)$. Poor patient prognosis is associated with TP53 mutations in various sarcoma subtypes, such as gliosarcoma (45), osteosarcoma (46), Ewing's sarcoma (47), chondrosarcoma (48) and liposarcoma (49). In the present study, the highly frequent TP53 mutations were identified in osteosarcoma, fibrosarcoma, liposarcoma and leiomyosarcoma, suggesting an association with poor prognosis in these subtypes.

Molecular diagnosis based on NGS detection can accurately characterize sarcomas according to molecular characteristics, which is a powerful complement to histological identification (50) since pathologists often provide descriptions such as 'probable' or 'possible' during sarcoma diagnosis. The molecular features of different sarcoma subtypes have been extensively studied. For example, $P D G F B$ rearrangement in DFSP, $M D M 2$ and $C D K 4$ amplification in liposarcoma, EWSR1 translocation in Ewing's sarcoma, and SS18 translocation in synovial sarcoma (51-54). With the additional assistance of NGS detection, 15 sarcomas that were difficult diagnose by histological examination were further classified. Notably, one misclassified sarcoma subtype was also successfully corrected. These results suggest that NGS technology can effectively assist in the diagnosis and classification of sarcoma subtypes. However, 54 cases were still not well classified, which may be due to the fact that the corresponding molecular characteristics or biomarkers of sarcoma are still not clearly understood. Therefore, the identification of sarcoma biomarkers is important for further diagnostic advancements.
A number of specific mutations have been used for the classification of sarcoma. For example, Pierron et al (55) defined a novel type of bone sarcoma by identifying the BCOR-CCNB3 gene fusion. Yoshida et al (56) identified that CIC-rearranged sarcomas were distinctly different from Ewing's sarcomas, clinically, morphologically and immunohistochemically. Furthermore, Michal et al (57) reported a EWSR1-SMAD3-rearranged fibroblastic tumor that represented a novel subtype, and Chiang et al (58) identified a novel tumor type with the features of fibrosarcoma by NTRK fusion. However, few reports have focused on the association between gene mutations and different sarcoma subtypes. In the present study, the associations between GAs and tumor subtypes, patient demographics and TMB values were analyzed, which may provide potential biomarkers for the future diagnosis of sarcoma.

KIT mutations are a significant phenotypic feature of GISTs (59). As predicted, the association between KIT mutations and GISTs was also identified in the present study. In addition, a significant negative association was observed between TP53 mutations and GISTs. These results suggest that mutations in both KIT and TP53 may be used as biomarkers for GIST diagnosis.

In osteosarcoma, the frequent mutation of $R B 1$ was highly prevalent, and was thus proposed as a potential prognostic biomarker (60). With the exception $R B 1$, the association between NCOR1 mutation and osteosarcoma was also identified in the present study. NCORl is a transcription factor that regulates various biological functions (61). As a tumor suppressor gene, mutation in NCORl was confirmed to be associated with the prognostic prediction of numerous cancers, such as breast cancer, 
Table IV. Association between mutated genes and sarcoma subtypes.

\begin{tabular}{|c|c|c|c|c|}
\hline Sarcoma subtype & Mutated gene & $\begin{array}{c}\text { Mutation frequency } \\
\text { within subtype, } \%\end{array}$ & $\begin{array}{l}\text { Mutation frequency } \\
\text { outside of subtype, } \%\end{array}$ & P-value \\
\hline \multirow[t]{19}{*}{ Osteosarcoma } & NCOR1 & 36.36 & 1.69 & $8.14 \times 10^{-7}$ \\
\hline & GID4 & 22.73 & 1.13 & $1.92 \times 10^{-4}$ \\
\hline & $L R P 1 B$ & 22.73 & 1.69 & $4.75 \times 10^{-4}$ \\
\hline & $R B 1$ & 31.82 & 6.21 & $1.12 \times 10^{-3}$ \\
\hline & $A U R K B$ & 13.64 & 0.00 & $1.19 \times 10^{-3}$ \\
\hline & $G L I 2$ & 13.64 & 0.00 & $1.19 \times 10^{-3}$ \\
\hline & RICTOR & 18.18 & 1.13 & $1.15 \times 10^{-3}$ \\
\hline & $M A P 2 K 4$ & 18.18 & 2.82 & $9.90 \times 10^{-3}$ \\
\hline & STK24 & 9.09 & 0.00 & 0.012 \\
\hline & TNFSF $13 B$ & 9.09 & 0.00 & 0.012 \\
\hline & CCNE1 & 13.64 & 1.69 & 0.019 \\
\hline & $P R K D C$ & 13.64 & 1.69 & 0.019 \\
\hline & PTEN & 22.73 & 6.21 & 0.020 \\
\hline & CCND3 & 18.18 & 3.95 & 0.022 \\
\hline & FGF 10 & 13.64 & 2.26 & 0.031 \\
\hline & BRD4 & 9.09 & 0.56 & 0.033 \\
\hline & PRKACA & 9.09 & 0.56 & 0.033 \\
\hline & $R E T$ & 9.09 & 0.56 & 0.033 \\
\hline & $I L 7 R$ & 13.64 & 2.82 & 0.046 \\
\hline \multirow[t]{10}{*}{ Myxofibrosarcoma } & TP53 & 91.67 & 31.02 & $4.28 \times 10^{-5}$ \\
\hline & $A K T 2$ & 25.00 & 0.53 & $6.57 \times 10^{-4}$ \\
\hline & FAM135B & 33.33 & 2.67 & $8.32 \times 10^{-4}$ \\
\hline & $C D K N 2 A$ & 58.33 & 16.04 & $1.77 \times 10^{-3}$ \\
\hline & $J U N$ & 25.00 & 1.60 & $3.06 \times 10^{-3}$ \\
\hline & $C D K N 2 B$ & 50.00 & 12.83 & $3.48 \times 10^{-3}$ \\
\hline & ROS1 & 16.67 & 1.07 & 0.019 \\
\hline & $A X L$ & 16.67 & 1.60 & 0.030 \\
\hline & SETD2 & 16.67 & 1.60 & 0.030 \\
\hline & CCNE1 & 16.67 & 2.14 & 0.044 \\
\hline \multirow[t]{3}{*}{ Leiomyosarcoma } & $M A P 2 K 4$ & 30.77 & 2.69 & $1.17 \times 10^{-3}$ \\
\hline & TP53 & 69.23 & 32.26 & 0.013 \\
\hline & KDM6A & 15.38 & 1.08 & 0.022 \\
\hline \multirow[t]{2}{*}{ GIST } & $K I T$ & 91.30 & 1.70 & $4.00 \times 10^{-23}$ \\
\hline & TP53 & 0.00 & 39.20 & $3.36 \times 10^{-5}$ \\
\hline \multirow[t]{8}{*}{ Liposarcoma } & $C D K 4$ & 64.29 & 3.78 & $1.72 \times 10^{-8}$ \\
\hline & $M D M 2$ & 64.29 & 4.86 & $6.96 \times 10^{-8}$ \\
\hline & $F R S 2$ & 50.00 & 4.32 & $7.70 \times 10^{-6}$ \\
\hline & FUS & 21.43 & 0.00 & $2.81 \times 10^{-4}$ \\
\hline & $L R P 1$ & 28.57 & 3.24 & $2.58 \times 10^{-3}$ \\
\hline & $M Y B$ & 14.29 & 0.00 & $4.62 \times 10^{-3}$ \\
\hline & PTPN11 & 14.29 & 0.54 & 0.013 \\
\hline & $T Y K 2$ & 14.29 & 1.62 & 0.041 \\
\hline \multirow[t]{5}{*}{ Synovial sarcoma } & SS18 & 77.78 & 0.00 & $1.63 \times 10^{-11}$ \\
\hline & TP53 & 0.00 & 36.32 & 0.029 \\
\hline & CREB3L1 & 11.11 & 0.00 & 0.045 \\
\hline & $P D K 1$ & 11.11 & 0.00 & 0.045 \\
\hline & TET1 & 11.11 & 0.00 & 0.045 \\
\hline \multirow[t]{4}{*}{ Ewing's sarcoma } & EWSR1 & 71.43 & 1.04 & $1.75 \times 10^{-7}$ \\
\hline & EPHB1 & 14.29 & 0.00 & 0.035 \\
\hline & $F E V$ & 14.29 & 0.00 & 0.035 \\
\hline & VGLL3 & 14.29 & 0.00 & 0.035 \\
\hline
\end{tabular}



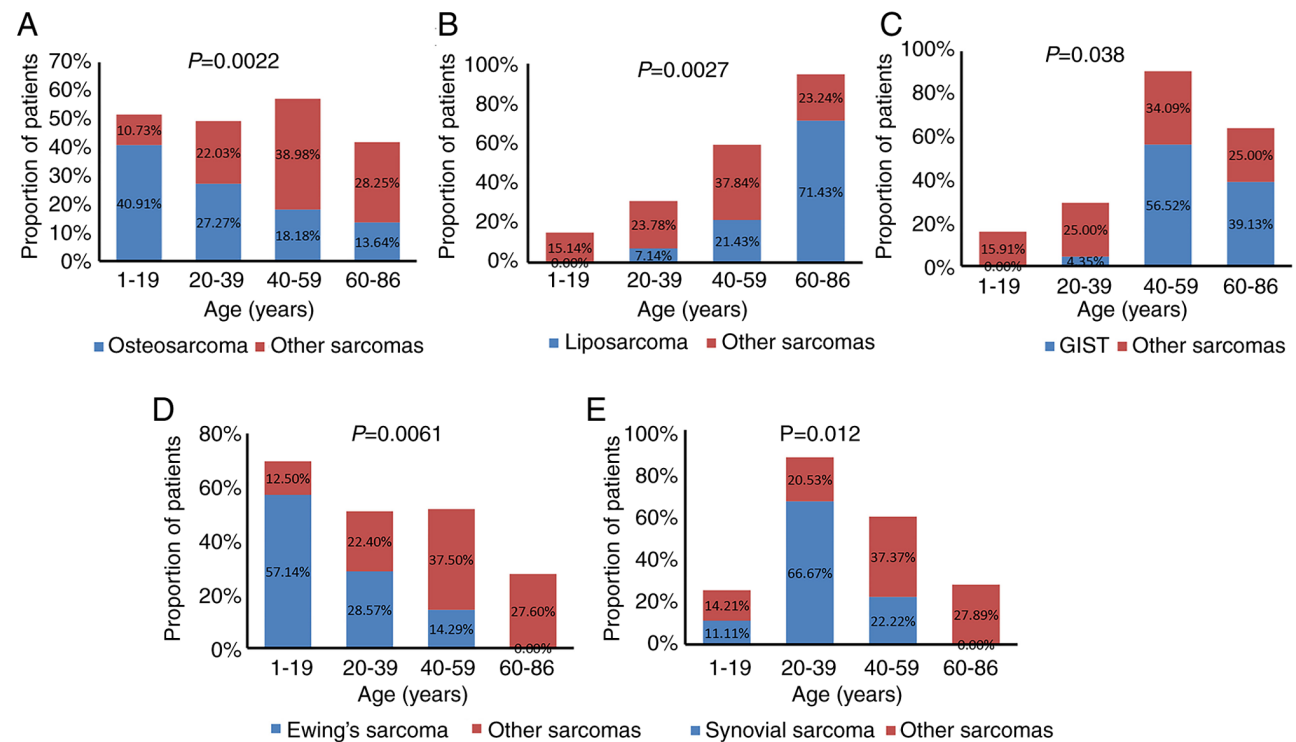

Figure 4. Association between patient age and sarcoma subtype for (A) osteosarcomas, (B) liposarcomas, (C) GISTs, (D) Ewing's sarcomas and (E) and synovial sarcomas. GIST, gastrointestinal stromal tumor.

A

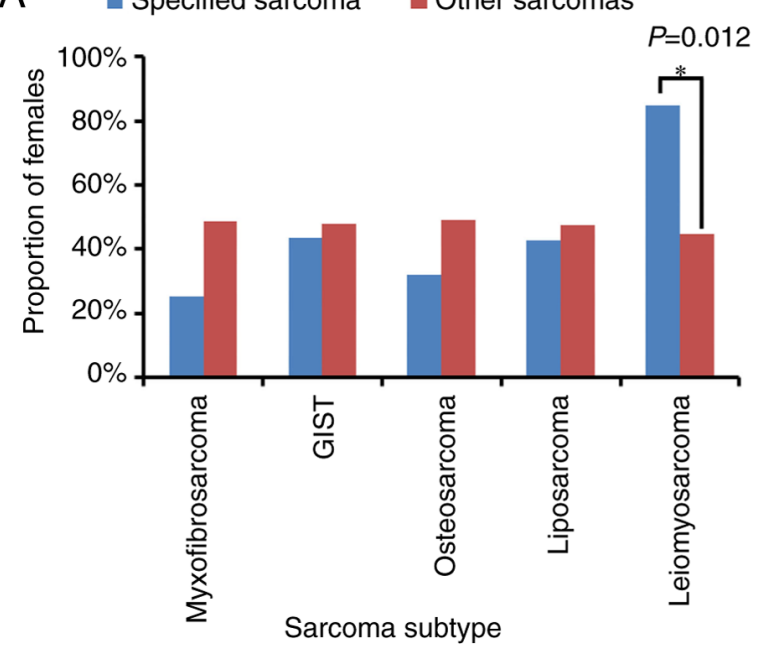

B

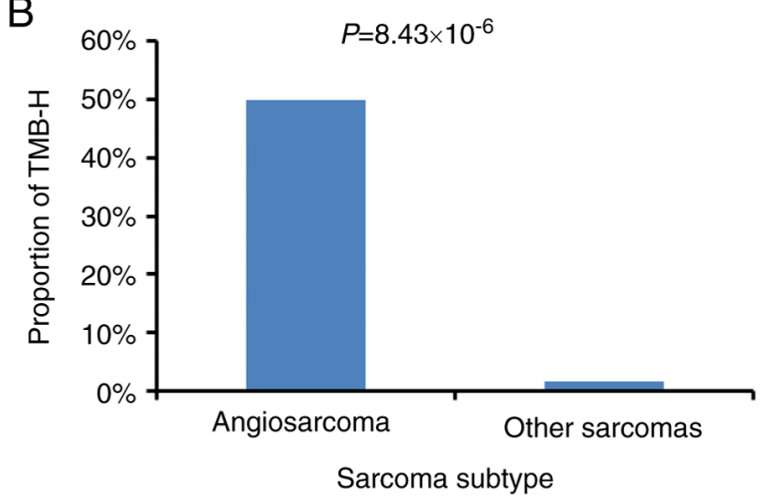

Figure 5. Association of sarcoma subtype with patient sex and TMB-H. (A) Association between specific sarcoma subtypes and the proportion of female patients. Each subtype (blue) was compared with the rest of the sarcoma subtypes (red). Leiomyosarcoma was the only subtype to be significantly associated with female patients; $\mathrm{P}=0.012$. (B) Association between TMB-H and angiosarcoma compared with the association between TMB-H and other sarcoma subtypes. TMB-H, tumor mutational burden $>10$ muts/Mb; GIST, gastrointestinal stromal tumor.

lung adenocarcinoma and GISTs $(62,63)$. These findings suggest that NCOR 1 mutations are a potential biomarker for the molecular diagnosis and prognosis of osteosarcoma. In the present study, genes such as GID4, LRPIB and PTEN were found to be significantly associated with osteosarcoma. These results have important relevance for guiding the diagnosis of osteosarcoma.

The amplification of $M D M 2$ and $C D K 4$ has been reported to occur in liposarcoma, and may therefore be considered as therapeutic targets (64), as well as used to assist the diagnosis of well-differentiated and dedifferentiated liposarcomas (65). The significant association between $C D K 4$ and MDM2 amplification and liposarcoma was detected in the present study, and was able to successfully classify 2 cases of liposarcoma from soft-tissue sarcomas. These results support the significance of NGS detection in the diagnosis of liposarcoma. In addition to $C D K 4$ and $M D M 2,6$ additional mutated genes (including FRS2, FUS, LRP1, MYB, PTPN11 and TYK2) were also associated with the liposarcoma subtype, indicating the potential diagnostic value of these genes in liposarcoma.

Fibrosarcoma can also be divided into multiple subtypes, such as myxofibrosarcoma, DFSP, solitary fibrous tumor and infantile fibrosarcoma. COL1A1-PDGFB fusion is a prominent molecular feature of DFSP (66). Mutations within the telomerase reverse transcriptase promoter were reported to be associated with the histologically malignant features of solitary fibrous tumors, and to some extent, to play an auxiliary role in their diagnosis and treatment (67). Although there are high mutational frequencies of TP53, RB1, CDKN2A, CDKN2B, NF1 and $N T R K 1$, few molecular predictors of myxofibrosarcoma have been identified (68). Due to the limited number of subtypes 
Table V. List of NTRK fusions in the present cohort.

\begin{tabular}{clll}
\hline Case & Gene & Mutation type & \multicolumn{1}{c}{ DNA change } \\
\hline 1 & NTRK1 & CNV & Gene amplification \\
2 & NTRK1 & CNV & Gene amplification \\
3 & NTRK1 & CNV & Gene amplification \\
4 & NTRK1 & CNV & Gene amplification \\
5 & NTRK3 & CNV & Gene amplification \\
6 & NTRK1 & FUSION & LMNA-NTRK1 \\
7 & NTRK1 & FUSION & IRF2BP2-NTRK1 \\
8 & NTRK3 & FUSION & MEF2A-NTRK3 \\
9 & NTRK3 & FUSION & ITFG1-NTRK3 \\
10 & NTRK3 & FUSION & ETV6-NTRK3 \\
11 & NTRK3 & FUSION & ETV6-NTRK3 \\
\hline
\end{tabular}

NTRK, tropomyosin-related kinase; CNV, copy number variation.

across the samples, only the association between the mutated genes and myxofibrosarcoma was analyzed in the present study, and the results showed that mutations in TP53, AKT2, FAM135B, CDKN2A, JUN, CDKN2B, ROS1, AXL, SETD2 and CCNE1 were significantly associated with myxofibrosarcoma. These results may be helpful for the diagnosis of myxofibrosarcoma. Although the number of cases was not large, the association between mutated genes and sarcoma subtype may still be used to guide molecular diagnoses. For example, based on 9 cases, a positive association was detected between the SS18 mutation and synovial sarcoma, and based on 7 cases, a significant association was also detected between the EWSR 1 mutation and Ewing's sarcoma. However, studies with larger cohorts are required to identify potential biomarkers for the auxiliary diagnosis of sarcomas.

The incidence rate of different sarcoma subtypes varies with sex and age. Classical osteosarcoma and rhabdomyosarcoma frequently occur in children and adolescents, while myxofibrosarcoma, synovial sarcoma, angiosarcoma, DFSP and clear cell sarcoma are more common in patients $>20$ years of age $(69,70)$. Also, myxofibrosarcoma, rhabdomyosarcoma and synovial sarcoma may be more likely to occur in men, while the occurrence of leiomyosarcoma was notably more common in female participants (70). The results of the present study support previous studies suggesting that osteosarcomas, Ewing's sarcomas, GISTs and liposarcomas are associated with patient age, and that leiomyosarcoma is associated with patient sex.

TMB is a novel biomarker for the prognosis of cancer patients treated with immune checkpoint inhibitors (ICPIs) $(71,72)$. The majority of sarcomas (such as osteosarcomas, GISTs and Ewing's sarcomas) are reported to have a low TMB $(73,74)$, while Trabucco (75) reported TMB-H in skin atypical fibroxanthoma and skin sarcoma. However, the results of the present study support that with the exception of 2 angiosarcoma cases, the TMB value of most sarcomas is low. Though only 4 cases were included in the current cohort, a significant association was detected between angiosarcomas and TMB-H, indicating that patients with angiosarcomas may benefit from ICPI therapy.
NTRK functions in the development, differentiation and metabolism of nerves and other tissues. NTRK inhibitors can be used as targeted agents for tumor therapy, thus the detection of NTRK fusions has important clinical significance (76-78). ETV6-NTRK3 fusion is common in infantile fibrosarcoma, and the tropomyosin-related kinase inhibitor LOXO-101 was reported to benefit infantile fibrosarcoma patients harboring ETV6-NTRK3 fusions (78). A metastatic infantile fibrosarcoma patient harboring LMNA-NTRK1 showed a complete and durable response to crizotinib (79). Furthermore, Wong et al (77) presented a case of a reverse transcription PCR ETV6-NTRK3-negative congenital infantile fibrosarcoma harboring a LMNA-NTRK1 gene fusion with a near-complete response to crizotinib. The data support the assumption that NTRK fusions are the drug target of LOXO-101 or crizotinib in sarcomas. In the present study, ETV6-NTRK3 and LMNA-NTRK1 fusions were successfully detected, indicating a potential treatment target for these patients. Follow-up information on the targeted treatment of patients with new NTRK fusions of IRF2BP2-NTRK1, MEF $2 A-N T R K 3$ and ITFG1-NTRK 3 may also guide and expand the use of NTRK fusion therapy in patients with sarcoma.

In conclusion, the present study investigated the genomic mutation profiles of pan-sarcomas, identified potential biomarkers, and accurately classified sarcoma subtypes with the assistance of NGS. The identification of NTRK fusions in sarcoma provides important value for NTRK inhibitor therapy. The absence of FISH confirmation is a limitation of the present study. However, the results support that NGS targeting may effectively promote the accurate classification and diagnosis of sarcomas, and provide guidance for precise therapeutic strategies for bone and soft-tissue sarcomas.

\section{Acknowledgements}

Not applicable.

\section{Funding}

No funding was received.

\section{Availability of data and materials}

The datasets used and/or analyzed in the current study are available from the corresponding author upon reasonable request.

\section{Authors' contributions}

LX, XX and JW recruited the patients, collected the samples, analyzed the data and wrote the manuscript; XS, PZ and AL processed samples, conducted experiments, analyzed data and reviewed the manuscript; $X S$ and $\mathrm{PZ}$ are responsible for the authenticity of the raw data. BZ designed and supervised the study. All authors read and approved the final manuscript.

\section{Ethical approval and consent to participate}

The present study was approved by the Ethics Committees of the National Cancer Center/Cancer Hospital, Chinese Academy of Medical Sciences and Peking Union Medical 
College and the First Affiliated Hospital of Sun Yat Sen University. Written informed consent for participation was obtained from all subjects.

\section{Patient consent for publication}

Not applicable.

\section{Competing interests}

The authors declare that they have no competing interests.

\section{References}

1. Taylor BS, Barretina J, Maki RG, Antonescu CR, Singer S and Ladanyi M: Advances in sarcoma genomics and new therapeutic targets. Nat Rev Cancer 11: 541-557, 2011.

2. Jemal A, Siegel R, Xu J and Ward E: Cancer statistics, 2010. CA Cancer J Clin 60: 277-300, 2010.

3. Szkandera J, Absenger G, Liegl-Atzwanger B, Pichler M, Stotz M, Samonigg H, Glehr M, Zacherl M, Stojakovic T, Gerger A and Leithner A: Elevated preoperative neutrophil/lymphocyte ratio is associated with poor prognosis in soft-tissue sarcoma patients. Br J Cancer 108: 1677-1683, 2013.

4. Chan JY, Zhang Z, Chew W, Tan GF, Lim CL, Zhou L, Goh WL, Poon E, Somasundaram N, Selvarajan S, et al: Biological significance and prognostic relevance of peripheral blood neutrophil-to-lymphocyte ratio in soft tissue sarcoma. Sci Rep 8: 11959, 2018.

5. Somasundaram N, Chan SH, Quek R and Ngeow J: Chapter 43-Advances in Sarcoma Genomics and Therapeutic Management. In: Oncogenomics. Dammacco F and Silvestris F, (eds.) Academic Press, pp609-621, 2019.

6. Penel N, Ray-Coquard I, Bal-Mahieu C, Chevreau C, Le Cesne A, Italiano A, Bompas E, Clisant S, Baldeyrou B, Lansiaux A, et al: Low level of baseline circulating VEGF-A is associated with better outcome in patients with vascular sarcomas receiving sorafenib: An ancillary study from a phase II trial. Target Oncol 9: 273-277, 2014.

7. Serranogarcia C, Heinrich MC, Zhu M, Raut CP, Eilers G, Ravegnini G, Demetri GD, Bauer S, Fletcher JA and George S: In vitro and in vivo activity of regorafenib (REGO) in drug-resistant gastrointestinal stromal tumors (GIST). J Clin Oncol 31 (Suppl 15): 10510-10510, 2013.

8. Rickel K, Fang F and Tao J: Molecular genetics of osteosarcoma. Bone 102: 69-79, 2017.

9. Siegel RL, Miller KD and Jemal A: Cancer statistics, 2016. CA Cancer J Clin 66: 7-30, 2016.

10. Maguire M: Comment on: Early symptoms of bone and soft tissue sarcomas: Could they be diagnosed earlier? Ann R Coll Surg Engl 94: 451, 2012.

11. Scurr M and Judson I: Sarcoma. Cancer Chemother Biol Response Modif 21: 637-653, 2003.

12. Hastings CA, Torkildson JC and Agrawal AK: Sarcomas of the Soft Tissues and Bone. In: Handbook of Pediatric Hematology and Oncology: Children's Hospital \& Research Center Oakland, Second edition, pp183-192, 2012.

13. Sinha S and Peach AH: Diagnosis and management of soft tissue sarcoma. BMJ 341: c7170, 2010.

14. Whelan J, McTiernan A, Cooper N, Wong YK, Francis M, Vernon S and Strauss SJ: Incidence and survival of malignant bone sarcomas in England 1979-2007. Int J Cancer 131: E508-E517, 2012.

15. Yang L, Chen Y, Cui T, Knösel T, Zhang Q, Geier C, Katenkamp D and Petersen I: Identification of biomarkers to distinguish clear cell sarcoma from malignant melanoma. Hum Pathol 43: 1463-1470, 2012.

16. van Brummelen EMJ, de Boer A, Beijnen JH and Schellens JHM: BRAF mutations as predictive biomarker for response to Anti-EGFR monoclonal antibodies. Oncologist 22: 864-872, 2017.

17. Evola FR, Costarella L, Pavone V, Caff G, Cannavò L, Sessa A, Avondo S and Sessa G: Biomarkers of osteosarcoma, chondrosarcoma, and ewing sarcoma. Front Pharmacol 8: 150, 2017.
18. Hutanu D, Popescu R, Stefanescu H, Pirtea L, Candea A, Sarau C, Boruga O, Mehdi L, Ciuca I and Tanasescu S: The molecular genetic expression as a novel biomarker in the evaluation and monitoring of patients with osteosarcoma-subtype bone cancer disease. Biochem Genet 55: 291-299, 2017.

19. Cancer Genome Atlas Research Network; Weinstein JN, Collisson EA, Mills GB, Shaw KR, Ozenberger BA, Ellrott K, Shmulevich I, Sander C and Stuart JM: The cancer genome atlas Pan-cancer analysis project. Nat Genet 45: 1113-1120, 2013.

20. Cancer Genome Atlas Research Network; Kandoth C, Schultz N, Cherniack AD, Akbani R, Liu Y, Shen H, Robertson AG, Pashtan I, Shen R, et al: Integrated genomic characterization of endometrial carcinoma. Nature 497: 67-73, 2013.

21. Cancer Genome Atlas Research Network, Electronic address: elizabeth.demicco@sinaihealthsystem.ca, et al: Comprehensive and integrated genomic characterization of adult soft tissue sarcomas. Cell 171: 950-965.e28, 2017.

22. Chudasama P, Mughal SS, Sanders MA, Hübschmann D, Chung I, Deeg KI, Wong SH, Rabe S, Hlevnjak M, Zapatka M, et al: Integrative genomic and transcriptomic analysis of leiomyosarcoma. Nat Commun 9: 144, 2018.

23. Mertens F, Antonescu CR and Mitelman F: Gene fusions in soft tissue tumors: Recurrent and overlapping pathogenetic themes. Genes Chromosomes Cancer 55: 291-310, 2016.

24. Italiano A, Bianchini L, Gjernes E, Keslair F, Ranchere-Vince D, Dumollard JM, Haudebourg J, Leroux A, Mainguené C, Terrier P, et al: Clinical and biological significance of CDK4 amplification in well-differentiated and dedifferentiated liposarcomas. Clin Cancer Res 15: 5696-5703, 2009.

25. Chibon F, Lagarde P, Salas S, Pérot G, Brouste V, Tirode F Lucchesi C, de Reynies A, Kauffmann A, Bui B, et al: Validated prediction of clinical outcome in sarcomas and multiple types of cancer on the basis of a gene expression signature related to genome complexity. Nat Med 16: 781-787, 2010.

26. Sankar S and Lessnick SL: Promiscuous partnerships in Ewing's sarcoma. Cancer Genet 204: 351-365, 2011.

27. Fligman I, Lonardo F, Jhanwar SC, Gerald WL, Woodruff J and Ladanyi M: Molecular diagnosis of synovial sarcoma and characterization of a variant SYT-SSX2 fusion transcript. Am J Pathol 147: 1592-1599, 1995.

28. Antonescu CR, Tschernyavsky SJ, Woodruff JM, Jungbluth AA Brennan MF and Ladanyi M: Molecular diagnosis of clear cell sarcoma: Detection of EWS-ATF1 and MITF-M transcripts and histopathological and ultrastructural analysis of 12 cases. J Mol Diagn 4: 44-52, 2002.

29. Kubota T: Gastrointestinal stromal tumor (GIST) and imatinib. Int J Clin Oncol 11: 184-189, 2006.

30. Stacchiotti S, Crippa F, Messina A, Pilotti S, Gronchi A, Blay JY and Casali PG: Response to imatinib in villonodular pigmented synovitis (PVNS) resistant to nilotinib. Clin Sarcoma Res 3: 8, 2013.

31. Rausch JL, Boichuk S, Ali AA, Patil SS, Liu L, Lee DM, Brown MF, Makielski KR, Liu Y, Taguchi T, et al: Opposing roles of KIT and ABL1 in the therapeutic response of gastrointestinal stromal tumor (GIST) cells to imatinib mesylate. Oncotarget 8: 4471-4483, 2017

32. Dickson MA, Tap WD, Keohan ML, D'Angelo SP, Gounder MM, Antonescu CR, Landa J, Qin LX, Rathbone DD, Condy MM, et al: Phase II trial of the CDK4 inhibitor PD0332991 in patients with advanced CDK4-amplified well-differentiated or dedifferentiated liposarcoma. J Clin Oncol 31: 2024-2028, 2013.

33. Knezevich SR, McFadden DE, Tao W, Lim JF and Sorensen PH: A novel ETV6-NTRK3 gene fusion in congenital fibrosarcoma. Nat Genet 18: 184-187, 1998

34. Haller F, Knopf J, Ackermann A, Bieg M, Kleinheinz K, Schlesner M, Moskalev EA, Will R, Satir AA, Abdelmagid IE, et al: Paediatric and adult soft tissue sarcomas with NTRK1 gene fusions: A subset of spindle cell sarcomas unified by a prominent myopericytic/haemangiopericytic pattern. J Pathol 238: 700-710, 2016.

35. Gibault L, Pérot G, Chibon F, Bonnin S, Lagarde P, Terrier P, Coindre JM and Aurias A: New insights in sarcoma oncogenesis: A comprehensive analysis of a large series of 160 soft tissue sarcomas with complex genomics. J Pathol 223: 64-71, 2011.

36. Schaefer IM, Cote GM and Hornick JL: Contemporary sarcoma diagnosis, genetics, and genomics. J Clin Oncol 36: 101-110, 2018.

37. Krystel-Whittemore M, Taylor MS, Rivera M, Lennerz JK, Le LP, Dias-Santagata D, Iafrate AJ, Deshpande V, Chebib I, Nielsen GP, et al: Novel and established EWSR1 gene fusions and associations identified by next generation sequencing and fluorescence in-situ hybridization. Hum Pathol 93: 65-73, 2019. 
38. Liang X, Li Q, Xu B, Hu S, Wang Q, Li Y, Zong Y, Zhang S and $\mathrm{Li}$ C: Mutation landscape and tumor mutation burden analysis of Chinese patients with pulmonary sarcomatoid carcinomas. Int J Clin Oncol 24: 1061-1068, 2019.

39. Zheng B, Qu Y, Wang J, Shi Y and Yan W: Pathogenic and targetable genetic alterations in resected recurrent undifferentiated pleomorphic sarcomas identified by targeted next-generation sequencing. Cancer Genomics Proteomics 16: 221-228, 2019.

40. Nagy A, Bhaduri A, Shahmarvand N, Shahryari J, Zehnder JL, Warnke RA, Mughal T, Ali S and Ohgami RS: Next-generation sequencing of idiopathic multicentric and unicentric Castleman disease and follicular dendritic cell sarcomas. Blood Adv 2: 481-491, 2018

41. Nakashima R, Fujita M, Enomoto T, Haba T, Yoshino K, Wada H, Kurachi H, Sasaki M, Wakasa K, Inoue M, et al: Alteration of p16 and p15 genes in human uterine tumours. Br J Cancer 80 458-467, 1999 .

42. Toguchida J, Yamaguchi T, Ritchie B, Beauchamp RL, Dayton SH, Herrera GE, Yamamuro T, Kotoura Y, Sasaki MS Little JB, et al: Mutation spectrum of the p53 gene in bone and soft tissue sarcomas. Cancer Res 52: 6194-6199, 1992.

43. Quinlan DC, Davidson AG, Summers CL, Warden HE and Doshi HM: Accumulation of p53 protein correlates with a poor prognosis in human lung cancer. Cancer Res 52: 4828-4831, 1992

44. Li JP, Zhang XM, Zhang Z, Zheng LH, Jindal S and Liu YJ: Association of p53 expression with poor prognosis in patients with triple-negative breast invasive ductal carcinoma. Medicine (Baltimore) 98: e15449, 2019.

45. Cho SY, Park C, Na D, Han JY, Lee J, Park OK, Zhang C, Sung CO, Moon HE, Kim Y, et al: High prevalence of TP53 mutations is associated with poor survival and an EMT signature in gliosarcoma patients. Exp Mol Med 49: e317, 2017.

46. Tsuchiya T, Sekine K, Hinohara S, Namiki T, Nobori T and Kaneko Y: Analysis of the p16INK4, p14ARF, p15, TP53, and MDM2 genes and their prognostic implications in osteosarcoma and Ewing sarcoma. Cancer Genet Cytogenet 120: 91-98, 2000.

47. Huang HY, Illei PB, Zhao Z, Mazumdar M, Huvos AG Healey JH, Wexler LH, Gorlick R, Meyers P and Ladanyi M: Ewing sarcomas with p53 mutation or p16/p14ARF homozygous deletion: A highly lethal subset associated with poor chemoresponse. J Clin Oncol 23: 548-558, 2005.

48. Oshiro Y, Chaturvedi V, Hayden D, Nazeer T, Johnson M, Johnston DA, Ordóñez NG, Ayala AG and Czerniak B: Altered p53 is associated with aggressive behavior of chondrosarcoma: A long term follow-up study. Cancer 83: 2324-2334, 1998.

49. Antonescu CR, Tschernyavsky SJ, Decuseara R, Leung DH, Woodruff JM, Brennan MF, Bridge JA, Neff JR, Goldblum JR and Ladanyi M: Prognostic impact of P53 status, TLS-CHOP fusion transcript structure, and histological grade in myxoid liposarcoma: A molecular and clinicopathologic study of 82 cases. Clin Cancer Res 7: 3977-3987, 2001.

50. Italiano A, Di Mauro I, Rapp J, Pierron G, Auger N, Alberti L, Chibon F, Escande F, Voegeli AC, Ghnassia JP, et al: Clinical effect of molecular methods in sarcoma diagnosis (GENSARC): A prospective, multicentre, observational study. Lancet Oncol 17: 532-538, 2016

51. Llombart B, Monteagudo C, Sanmartin O, López-Guerrero JA, Serra-Guillén C, Poveda A, Jorda E, Fernandez-Serra A, Pellín A, Guillén C and Llombart-Bosch A: Dermatofibrosarcoma protuberans: A clinicopathological, immunohistochemical, genetic (COL1A1-PDGFB), and therapeutic study of low-grade versus high-grade (fibrosarcomatous) tumors. J Am Acad Dermatol 65: $564-575,2011$.

52. Kammerer-Jacquet SF, Thierry S, Cabillic F, Lannes M, Burtin F, Henno S, Dugay F, Bouzillé G, Rioux-Leclercq N, Belaud-Rotureau MA and Stock N: Differential diagnosis of atypical lipomatous tumor/well-differentiated liposarcoma and dedifferentiated liposarcoma: Utility of p16 in combination with MDM2 and CDK4 immunohistochemistry. Hum Pathol 59: 34-40, 2017.

53. Kim SK and Park YK: Ewing sarcoma: A chronicle of molecular pathogenesis. Hum Pathol 55: 91-100, 2016

54. El Beaino M, Araujo DM, Lazar AJ and Lin PP: Synovial sarcoma: Advances in diagnosis and treatment identification of new biologic targets to improve multimodal therapy. Ann Surg Oncol 24: 2145-2154, 2017.

55. Pierron G, Tirode F, Lucchesi C, Reynaud S, Ballet S, Cohen-Gogo S, Perrin V, Coindre JM and Delattre O: A new subtype of bone sarcoma defined by BCOR-CCNB3 gene fusion. Nat Genet 44: 461-466, 2012
56. Yoshida A, Goto K, Kodaira M, Kobayashi E, Kawamoto H, Mori T, Yoshimoto S, Endo O, Kodama N, Kushima R, et al: CIC-rearranged Sarcomas: A study of 20 cases and comparisons with Ewing sarcomas. Am J Surg Pathol 40: 313-323, 2016.

57. Michal M, Berry RS, Rubin BP, Kilpatrick SE, Agaimy A, Kazakov DV, Steiner P, Ptakova N, Martinek P, Hadravsky L, et al: EWSR1-SMAD3-rearranged Fibroblastic Tumor: An Emerging Entity in an Increasingly More Complex Group of Fibroblastic/Myofibroblastic Neoplasms. Am J Surg Pathol 42: 1325-1333, 2018

58. Chiang S, Cotzia P, Hyman DM, Drilon A, Tap WD, Zhang L, Hechtman JF, Frosina D, Jungbluth AA, Murali R, et al: NTRK fusions define a novel uterine sarcoma subtype with features of fibrosarcoma. Am J Surg Pathol 42: 791-798, 2018.

59. Fletcher JA and Rubin BP: KIT mutations in GIST. Curr Opin Genet Dev 17: 3-7, 2007.

60. Ren W and Gu G: Prognostic implications of RB1 tumour suppressor gene alterations in the clinical outcome of human osteosarcoma: A meta-analysis. Eur J Cancer Care (Engl): 26, 2017 doi: $10.1111 /$ ecc.12401.

61. Müller L, Hainberger D, Stolz V, Hamminger P, Hassan H, Preglej T, Boucheron N, Sakaguchi S, Wiegers GJ, Villunger A, et al: The corepressor NCOR1 regulates the survival of single-positive thymocytes. Sci Rep 7: 15928, 2017.

62. Noblejas-Lopez MDM, Morcillo-Garcia S, Nieto-Jimenez C, Nuncia-Cantarero M, Győrffy B, Galan-Moya EM, Pandiella A and Ocaña A: Evaluation of transcriptionally regulated genes identifies NCOR1 in hormone receptor negative breast tumors and lung adenocarcinomas as a potential tumor suppressor gene. PLoS One 13: e0207776, 2018.

63. Wang W, Song XW, Bu XM, Zhang $\mathrm{N}$ and Zhao CH: PDCD2 and NCoR1 as putative tumor suppressors in gastric gastrointestinal stromal tumors. Cell Oncol (Dordr) 39: 129-137, 2016.

64. Kanojia D, Nagata Y, Garg M, Lee DH, Sato A, Yoshida K, Sato Y, Sanada M, Mayakonda A, Bartenhagen C, et al: Genomic landscape of liposarcoma. Oncotarget 6: 42429-42444, 2015.

65. Binh MB, Sastre-Garau X, Guillou L, de Pinieux G, Terrier P, Lagacé R, Aurias A, Hostein I and Coindre JM: MDM2 and CDK4 immunostainings are useful adjuncts in diagnosing well-differentiated and dedifferentiated liposarcoma subtypes: A comparative analysis of 559 soft tissue neoplasms with genetic data. Am J Surg Pathol 29: 1340-1347, 2005.

66. Zhang Z, Chen $\mathrm{H}$, Chen $\mathrm{M}$, He $\mathrm{X}$, Wang $\mathrm{Y}$ and Zhang $\mathrm{H}$ : Application of COL1A1-PDGFB fusion gene detection by fluorescence in situ hybridization in biopsy tissue of dermatofibrosarcoma protuberans. J Dermatol 44: 798-802, 2017.

67. Bianchi G, Sambri A, Pedrini E, Pazzaglia L, Sangiorgi L, Ruengwanichayakun P, Donati D, Benassi MS and Righi A: Histological and molecular features of solitary fibrous tumor of the extremities: Clinical correlation. Virchows Arch 476: 445-454, 2020

68. Ogura K, Hosoda F, Arai Y, Nakamura H, Hama N, Totoki Y, Yoshida A, Nagai M, Kato M, Arakawa E, et al: Integrated genetic and epigenetic analysis of myxofibrosarcoma. Nat Commun 9: 2765, 2018

69. Rogozhin DV, Bulycheva IV, Konovalov DM, Talalaev AG, Roshchin VY, Ektova AP, Bogoroditsky YS, Strykov VA, Kazakova AN, Olshanskaya YV, et al: Classical osteosarcoma in children and adolescent. Arkh Patol 77: 68-74, 2015 (In Russian).

70. Mans DR, Budhu Lall AE, Macnack VL, van Tholl JA Zandveld EB and Vrede MA: Incidence, and gender, age and ethnic distribution of sarcomas in the republic of Suriname from 1980 to 2008. West Indian Med J 63: 121-127, 2014.

71. Knepper TC, Montesion M, Russell JS, Sokol ES, Frampton GM, Miller VA, Albacker LA, McLeod HL, Eroglu Z, Khushalani NI, et al: The genomic landscape of Merkel cell carcinoma and clinicogenomic biomarkers of response to immune checkpoint inhibitor therapy. Clin Cancer Res 25: 5961-5971, 2019.

72. Chalmers ZR, Connelly CF, Fabrizio D, Gay L, Ali SM, Ennis R, Schrock A, Campbell B, Shlien A, Chmielecki J, et al: Analysis of 100,000 human cancer genomes reveals the landscape of tumor mutational burden. Genome Med 9: 34, 2017.

73. Cote GM, He J and Choy E: Next-generation sequencing for patients with sarcoma: A single center experience. Oncologist 23: 234-242, 2018

74. Chen X, Bahrami A, Pappo A, Easton J, Dalton J, Hedlund E, Ellison D, Shurtleff S, Wu G, Wei L, et al: Recurrent somatic structural variations contribute to tumorigenesis in pediatric osteosarcoma. Cell Rep 7: 104-112, 2014. 
75. Trabucco SE, Ali SM, Sokol E, Schrock AB, Albacker LA, Chung J, Xavier MF, Disel U, Miller VA, Ross JS, et al: Frequency of genomic biomarkers of response to immunotherapy in sarcoma. J Clin Oncol 36: 11579-11579, 2018.

76. Lange AM and Lo HW: Inhibiting TRK proteins in clinical cancer therapy. Cancers (Basel) 10: 105, 2018.

77. Wong V, Pavlick D, Brennan T, Yelensky R, Crawford J, Ross JS, Miller VA, Malicki D, Stephens PJ, Ali SM and Ahn H: Evaluation of a congenital infantile fibrosarcoma by comprehensive genomic profiling reveals an LMNA-NTRK1 gene fusion responsive to Crizotinib. J Natl Cancer Inst 108: djv307, 2016.

78. Nagasubramanian R, Wei J, Gordon P, Rastatter JC, Cox MC and Pappo A: Infantile fibrosarcoma with NTRK3-ETV6 fusion successfully treated with the tropomyosin-related kinase inhibitor LOXO-101. Pediatric Blood Cancer 63: 1468-1470, 2016.
79. Bender J, Anderson B, Bloom DA, Rabah R, McDougall R, Vats P and Mody R: Refractory and metastatic infantile fibrosarcoma harboring LMNA-NTRK1 fusion shows complete and durable response to crizotinib. Cold Spring Harb Mol Case Stud 5: a003376, 2019.

(i) (9) This work is licensed under a Creative Commons Attribution-NonCommercial-NoDerivatives 4.0 International (CC BY-NC-ND 4.0) License. 